\title{
Comment on "Incidence of Type 1 Diabetes among Children and Adolescents in Italy between 2009 and 2013: The Role of a Regional Childhood Diabetes Registry"
}

\author{
Paola Ballotari, ${ }^{1,2}$ Valeria Manicardi, ${ }^{3}$ and Paolo Giorgi Rossi ${ }^{1,2}$ \\ ${ }^{1}$ Interinstitutional Epidemiology Unit, Local Health Authority of Reggio Emilia, Via Amendola 2, 42122 Reggio Emilia, Italy \\ ${ }^{2}$ Arcispedale Santa Maria Nuova-IRCCS, Viale Umberto I 50, 42123 Reggio Emilia, Italy \\ ${ }^{3}$ Internal Medicine Department, Montecchio Hospital, Local Health Authority of Reggio Emilia, Via Barilla 16, 42027 \\ Montecchio, Italy
}

Correspondence should be addressed to Paola Ballotari; paola.ballotari@ausl.re.it

Received 15 July 2016; Accepted 25 August 2016

Academic Editor: Ulrike Rothe

Copyright (C) 2016 Paola Ballotari et al. This is an open access article distributed under the Creative Commons Attribution License, which permits unrestricted use, distribution, and reproduction in any medium, provided the original work is properly cited.

We read the article by Fortunato et al. [1] with great interest. In this study, one of the aims was to evaluate the incidence of Type 1 Diabetes (T1DM) in subjects $<18$ years of age in Apulia (Italy) by using three data sources: a hospital discharge registry, user fee exemption registry, and drug prescription registry. The authors found a progressive decrease in the annual incidence rate over the study period (2009-2013). According to previous studies, the incidence rate of Type 1 Diabetes among children (0-14 years of age) had been increasing [2-5] over the past 20 years. Moreover, a similar study carried out in Friuli-Venezia Giulia (a region in northern Italy) [6] found an almost stable incidence over the same period, excluding the first year of registration (2010). In the province of Reggio Emilia (Italy), where the diabetes register has been collecting data since 2009 [7], the trend of incidence is stable. Despite actions performed to clean up the Apulian data, residual misclassification of prevalent cases may have affected the data. Indeed, given the absence of a clinical database where the date of diagnosis is recorded, some prevalent cases may have been misclassified as incident cases; however, such misclassification declines after some years of registration because the undetected prevalent cases diminish. Therefore, the final effect of this declining bias is a false decrease of incident cases in the initial years of registration. Table 1, which is based on our data, reports the difference in incidence rate calculated using the date of diagnosis and the date of entry from the linkage of the data sources. A highly interesting approach for the Apulian register could be to broaden the network of pediatricians and/or to include hospital pediatric units in the same network, given their role in managing T1DM in children and adolescents. In this way, the register would merge clinical and routinely collected databases, thus enhancing completeness and reliability.

TABLE 1: T1DM incidence rates (per 100,000) among subjects, 0-18 years of age, in Reggio Emilia province, 2010-2013, by date of diagnosis and by date of entry.

\begin{tabular}{lccccccc}
\hline \multirow{2}{*}{ Year } & \multirow{2}{*}{ Population } & Cases & By date of diagnosis & \multicolumn{3}{c}{ By date of entry } \\
& & 16 & 16.7 & $95 \%$ CI & Cases & Rate & 22.0 \\
2009 & 95625 & 18 & 18.4 & $10.9-29.1$ & 21 & $13.6-33.6$ \\
2010 & 97798 & 16 & 16.1 & $9.2-26.2$ & 15 & 21.5 & 13.1 \\
2011 & 99217 & 19 & 19.5 & $11.2-30.4$ & 18 & 18.5 \\
2012 & 97473 & 17 & 17.1 & $10.0-27.4$ & 19 & 18.8 \\
2013 & 99249 & 19 & 18.8 & $11.3-29.4$ & 19 & 19.1 \\
2014 & 100815 & & &
\end{tabular}




\section{Competing Interests}

The authors declare that there is no conflict of interests regarding the publication of this paper.

\section{References}

[1] F. Fortunato, M. G. Cappelli, M. M. Vece et al., "Incidence of type 1 diabetes among children and adolescents in Italy between 2009 and 2013: the role of a regional childhood diabetes registry," Journal of Diabetes Research, vol. 2016, Article ID 7239692, 7 pages, 2016.

[2] G. Bruno, M. Maule, A. Biggeri et al., "More than 20 years of registration of type 1 diabetes in sardinian children: temporal variations of incidence with age, period of diagnosis, and year of birth," Diabetes, vol. 62, no. 10, pp. 3542-3546, 2013.

[3] J. G. B. Derraik, P. W. Reed, C. Jefferies, S. W. Cutfield, P. L. Hofman, and W. S. Cutfield, "Increasing incidence and age at diagnosis among children with type 1 diabetes mellitus over a 20 -year period in auckland (New zealand)," PLoS ONE, vol. 7, no. 2, Article ID e32640, 2012.

[4] V. Harjutsalo, L. Sjöberg, and J. Tuomilehto, "Time trends in the incidence of type 1 diabetes in Finnish children: a cohort study," The Lancet, vol. 371, no. 9626, pp. 1777-1782, 2008.

[5] J. H. Kim, C. G. Lee, Y. A. Lee, S. W. Yang, and C. H. Shin, "Increasing incidence of type 1 diabetes among Korean children and adolescents: analysis of data from a nationwide registry in Korea," Pediatric Diabetes, 2015.

[6] F. Valent, R. Candido, E. Faleschini et al., "The incidence rate and prevalence of pediatric type 1 diabetes mellitus (age 018) in the Italian region Friuli Venezia Giulia: populationbased estimates through the analysis of health administrative databases," Acta Diabetologica, vol. 53, no. 4, pp. 629-635, 2016.

[7] P. Ballotari, S. Chiatamone Ranieri, M. Vicentini et al., "Building a population-based diabetes register: an Italian experience," Diabetes Research and Clinical Practice, vol. 103, no. 1, pp. 7987, 2014. 


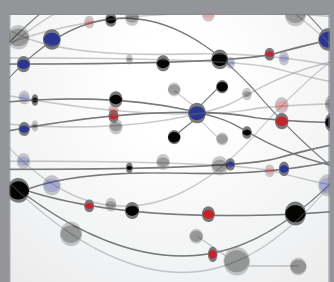

The Scientific World Journal
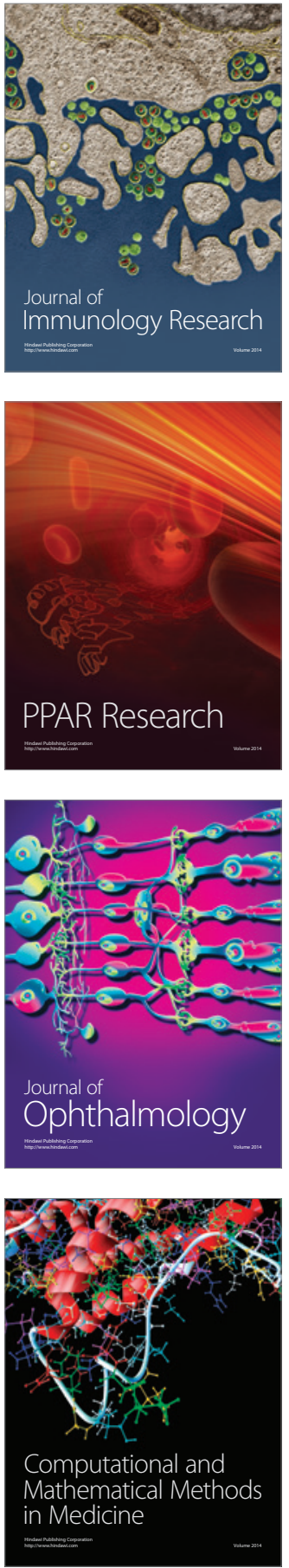

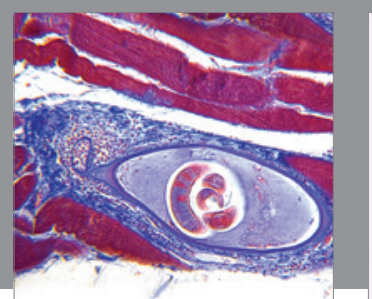

Gastroenterology Research and Practice

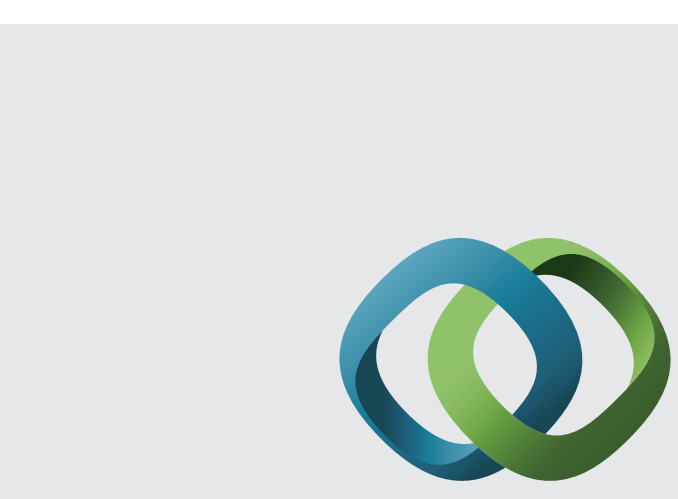

\section{Hindawi}

Submit your manuscripts at

http://www.hindawi.com
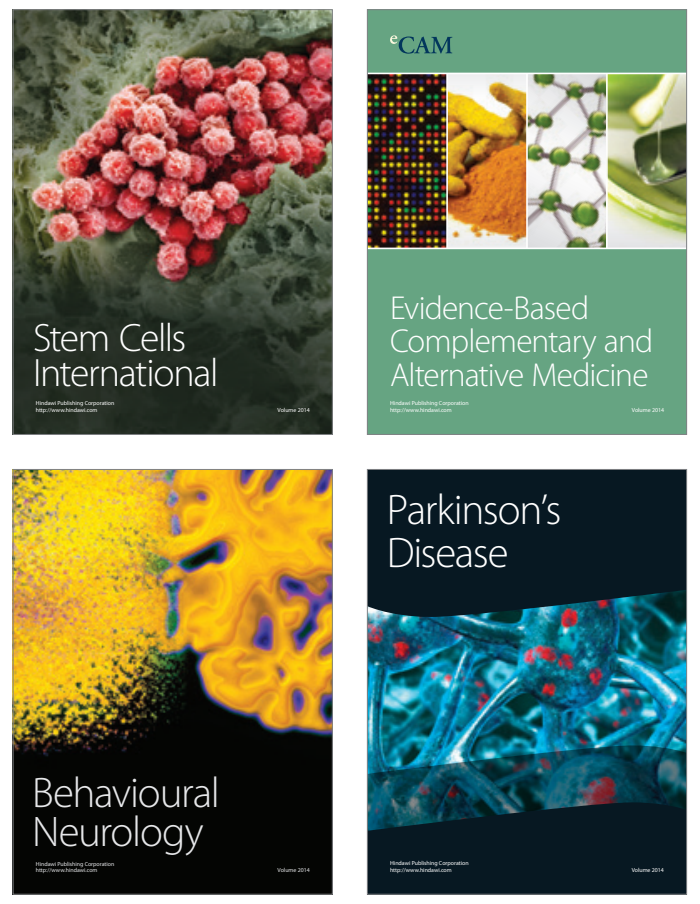
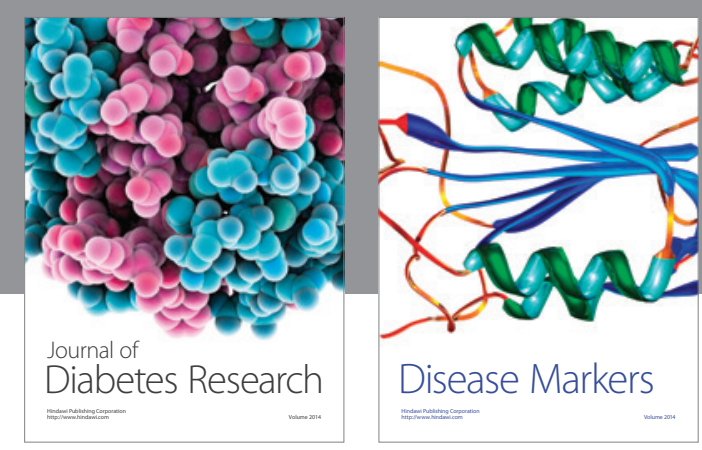

Disease Markers
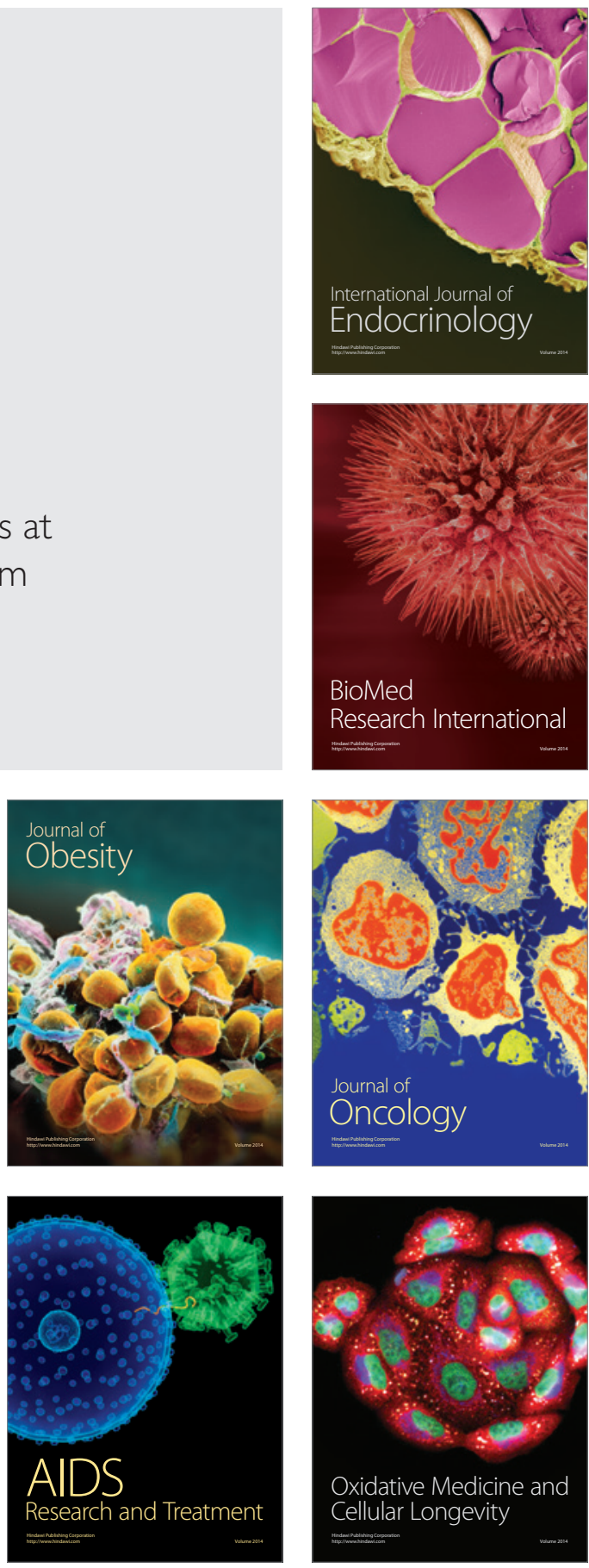